\title{
E-selectin in women with autoimmune thyroiditis and the relationships with metabolic syndrome
}

1Sieminska L., 2Walczak K., 3Borowski A., 1 Kos-Kudla B., 1 Marek B., 1 Nowak M., 1 Kajdaniuk D., 1 Foltyn W.

1Department of Pathophysiology and Endocrinology, Medical University of Silesia, Katowice, Poland 2Regional Railway Hospital, Katowice, Poland 3The Silesia Urology Centre Urovita, Chorzow, Poland

Autoimmune thyroiditis (AIT) is characterized by infiltration of the thyroid by lymphocytes and other immune cells. Autoantibodies to thyroid-specific antigens: thyroglobulin (TG) and thyroperoxidase (TPO) are also produced. The penetration of inflammatory cells into target organ is regulated by cytokines and adhesion molecules expressed on thyroid endothelial cells. E-selectin is one of the selectin family, and is responsible for interaction between leukocytes and endothelium and facilitates the accumulation of inflammation cells in thyroid gland. It is suggested that chronic inflammation and endothelial dysfunction can lead to increased cardiovascular risk and metabolic syndrome (MS) in AIT.

The aims of the study were:

to investigate whether AIT is associated with elevated serum levels of E-selectin, to examine relationships between E-selectin, thyroid function, and different risk factors - components of MS.

Materials and Methods: Two hundred fifty eight postmenopausal, euthyroid women were enrolled into the study. In 76 women AIT was recognized as the presence of elevated at least one of thyroid antibodies (TPO-Abs, TG-Abs) and the presence of typical thyroid sonography. One hundred eighty two women without AIT were control group. Anthropometric measurements were conducted (BMI, WHR) and blood pressure was measured. In all subjects were assessed: lipid profile, glucose, TSH, fT4, TPO-Ab, TG-Ab and E-selectin concentrations. Thyroid ultrasound scan was performed. The diagnosis of MS was performed using AHA/NHLBI criteria.

Results:

When we compared AIT women and control group there were no differences in serum E-selectin concentrations.

E-selectin was higher in MS for the two groups, but the prevalence of MS was similar.

When we analyzed all studied women, E-selectin positively correlated with BMI, WHR, and negatively with cHDL and fT4.

AIT was associated with elevated TSH.
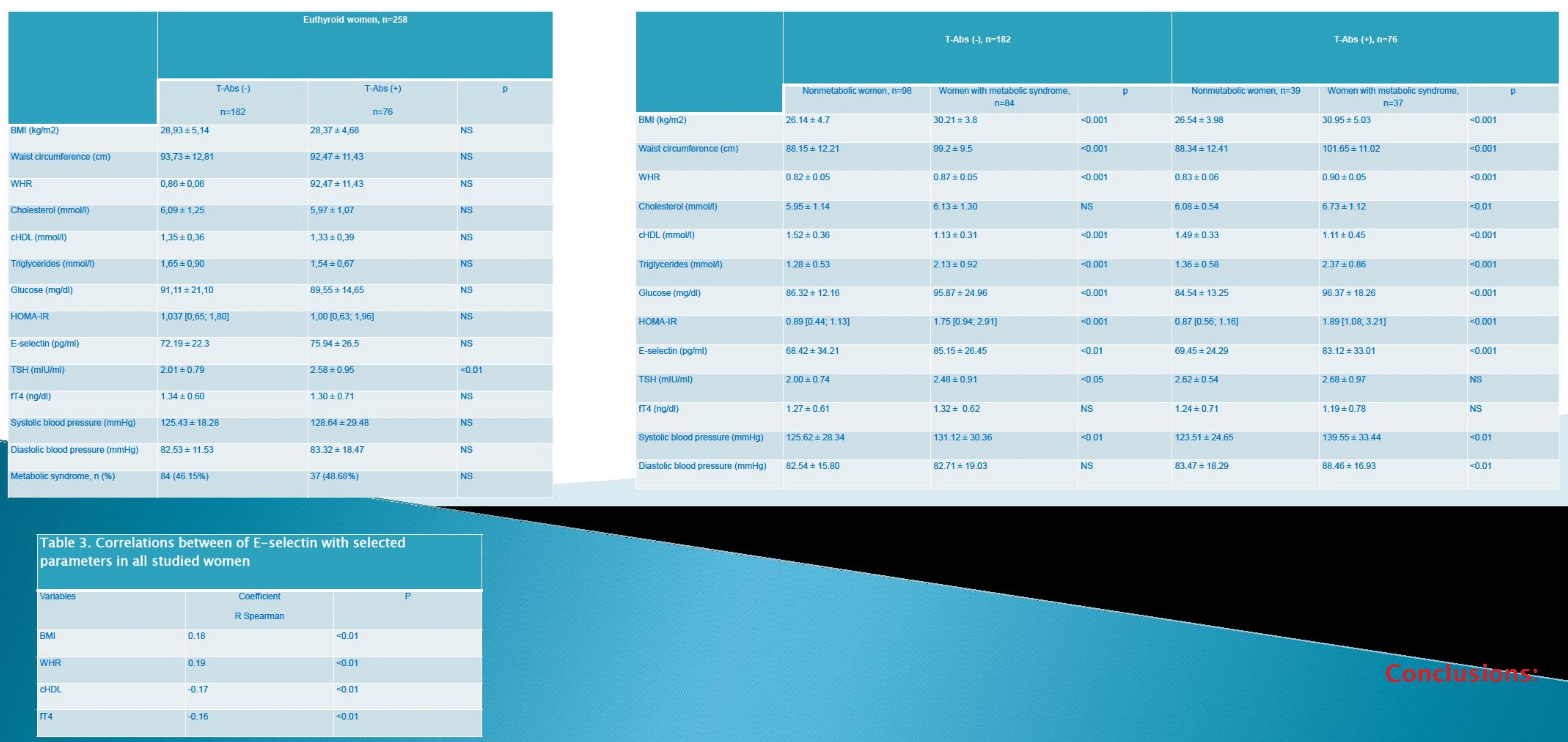